FORMATION Formation emploi

Revue française de sciences sociales

$110 \mid 2010$

Pêle-mêle

\title{
Mobilité géographique des jeunes : du système éducatif à l'emploi
}

Une approche coûts/bénéfices des distances parcourues

Geographical mobility of youth:from the educational system to employment

A Cost/Benefit Approach to the Distance Travelled

Geografische mobilität junger erwerbstätiger:vom bildungssystem zur

erwerbstätigkeit

Zurückgelegte Entfernungen nach einer Kosten/Nutzen-Betrachtung

Movilidad geográfica de los jóvenes: del sistema educativo al empleo

Un enfoque costo/beneficio de las distancias recorridas

\section{Philippe Lemistre et Marie-Benoît Magrini}

\section{(2) OpenEdition}

\section{Journals}

Édition électronique

URL : http://journals.openedition.org/formationemploi/3051

DOI : 10.4000/formationemploi.3051

ISSN : 2107-0946

Éditeur

La Documentation française

Édition imprimée

Date de publication : 15 juin 2010

Pagination : 63-78

ISSN : 0759-6340

Référence électronique

Philippe Lemistre et Marie-Benoît Magrini, «Mobilité géographique des jeunes : du système éducatif à l'emploi », Formation emploi [En ligne], 110 | 2010, mis en ligne le 10 juillet 2012, consulté le 30 octobre 2020. URL : http://journals.openedition.org/formationemploi/3051 ; DOI : https://doi.org/10.4000/ formationemploi.3051

(C) Tous droits réservés 


\section{Accès à l'emploi \\ et insertion}

\section{Mobilité géographique des jeunes: du système éducatif à l'emploi}

\section{Une approche coûts/bénéfices des distances parcourues}

Philippe Lemistre et Marie-Benoît Magrini*

Si la mobilité géographique des jeunes est différenciée selon le niveau de formation, elle dépend également du niveau de qualification de l'emploi, et des caractéristiques des territoires de départ et d'arrivée.

Parmi les objectifs de la stratégie de Lisbonne ${ }^{1}$, la promotion de la mobilité géographique des travailleurs a de nouveau été avancée comme un enjeu majeur pour un meilleur fonctionnement du marché du travail. Les analyses du chômage ne manquent pas également d'exposer qu'une plus grande mobilité des travailleurs améliorerait les appariements sur le marché du travail (Freyssinet, 2004). Pour autant, moins de $2 \%$ des citoyens de l'Union européenne vivent et travaillent dans un État membre autre que leur État d'origine, et cette proportion n'a guère évolué depuis trente ans (Green, Baldauf, Owen, 2009). Ainsi, malgré cette nouvelle recommandation en la matière, suite au Traité de Maastricht, la mobilité des travailleurs reste

\footnotetext{
1 La «stratégie de Lisbonne » désigne la politique économique de l'Union européenne entre 2000 et 2010, décidée au Conseil européen de Lisbonne en mars 2000 et retranscrite dans le traité de Lisbonne. Parmi les priorités affichées dans ce texte figure en bonne place la recherche d'une plus grande mobilité géographique des travailleurs et des étudiants.
}

avant tout une question infranationale et, plus précisément, une question très locale. En effet, si chaque année $7 \%$ des citoyens européens changent de lieu de résidence, plus de la moitié d'entre eux réalisent cette mobilité au sein de la même agglomération².

\footnotetext{
2 Source : «Les Européens face à la mobilité », Fenêtre sur l'Europe, 30 novembre 2006.
}

* Philippe Lemistre est ingénieur de recherche au Céreq, économiste au LIRHE (Laboratoire interdisciplinaire de recherche sur les ressources humaines et l'emploi). Ses travaux portent sur les carrières salariales et l'articulation entre système éducatif et marché du travail. Il a récemment publié : La formation initiale, une valeur sûre ? Presses de l'université de Toulouse Capitole, 2010. II a coordonné un ouvrage avec B. Conter et B. Reynes, L'ancienneté professionnelle à l'épreuve de la flexicurité, Presses de l'Université des Sciences Sociales, 2009 
Marie-Benoît Magrini est ingénieur de recherche à I'INRA (Institut national de recherche agronomique) Toulouse au sein de l'équipe loda (Innovations, organisations et dynamiques agro-industrielles) de I'UMR (unité mixte de recherche) 1248 Agir. Elle a soutenu, en 2006, une thèse en économie sur le rôle de la mobilité géographique sur l'insertion (université Toulouse I). Elle a récemment publié : "Les rendements de la mobilité spatiale des jeunes actifs», Revue d'économie régionale et urbaine, $n^{\circ} 3$. Elle a contribué à "Approches de la mobilité étudiante ", net.doc Céreq, sous la coordination du Groupe de Travail sur l'Enseignement Supérieur, 2009.

Cependant, au sein de la population, les jeunes actifs restent les individus les plus mobiles (Long, 1988 ; Pailhé et Solaz, 2001). Cette plus grande propension à la mobilité est probablement due à des contraintes familiales moins importantes, à un moindre attachement au territoire lié à un temps vécu sur ce dernier plus court (Da Vanzo, 1983). Bien souvent également, les jeunes ne sont pas encore propriétaires de leur logement alors que ce facteur est reconnu comme un frein important à la mobilité (Debrand et Taffin, 2005). Face à ces moindres contraintes, la mobilité spatiale peut être considérée, chez les jeunes, comme une stratégie d'insertion (Drapier, 2001). Les jeunes, en changeant d'espace, peuvent en effet améliorer la qualité de leur emploi, voire tout simplement accéder à l'emploi, et ils le font d'autant plus que les contraintes de mobilité sont moins fortes pour eux (Perret et Roux, 2004).

Pour autant, les chiffres sur la mobilité des jeunes témoignent, là aussi, que cette question reste avant tout infranationale. Selon les différentes études considérées ${ }^{3}$, entre $3 \%$ et $6 \%$ des jeunes diplômés français obtiennent leur premier emploi à l'étranger (ce taux atteint entre $10 \%$ et $12 \%$ pour les $3^{\text {es }}$ cycles scientifiques ou étudiants de grandes Écoles) et entre $1 \%$ et $4 \%$ y travaillent encore quatre ans après leur entrée dans la vie active ${ }^{4}$. En revanche, au niveau infranational, la mobilité des jeunes est plus importante et particulièrement si l'on considère une échelle repré-

\footnotetext{
${ }^{3}$ Sources : Études réalisées par les grandes Écoles sur leurs promotions d'élèves, Eurostat, enquête « Génération 98 », calculs des auteurs, Murdoch et Perret (2007).

${ }^{4}$ La moyenne européenne est respectivement de $4 \%$ et $3 \%$.
}

sentative de marchés locaux de travail, comme nous le verrons.

Pour étudier ces mobilités, la plupart des études révèlent une propension à la mobilité différenciée selon le niveau d'études (Drapier et Jayet, 2002 ; Yankow, 2003 ; Magrini, 2007). Or la qualification de l'emploi occupé s'avère tout aussi déterminante. En effet, si le niveau d'études demeure un élément essentiel de la qualification sur le marché du travail, le lien niveau d'études/niveau d'emploi se distend (Giret, Lopez et Rose, 2005). Une approche de la mobilité par l'emploi peut ainsi apparaître plus pertinente, d'une part au regard de la montée du phénomène de déclassement (Giret et Lemistre, 2004), et d'autre part car l'évolution de l'offre d'éducation a eu pour corollaire la création de nombreux diplômes conduisant au constat d'une valorisation différenciée des formations selon les spécialités de formation et/ ou la sélectivité des filières (Dauty, Lemistre, Vincens, 2006). Des jeunes ayant atteint un même niveau d'études peuvent alors s'engager dans des carrières professionnelles différentes, susceptibles d'induire différents comportements de mobilité. Il ne fait alors aucun doute que les déterminants de la mobilité sont différenciés, au moins autant en fonction de la qualification de l'emploi cible que du niveau d'études. Par exemple, certains bac +2 s'engagent dans une carrière de profession intermédiaire et d'autres dans un parcours d'employé (Béduwé C. et Planas J., 2001).

Nous proposons donc de retenir des groupes d'individus selon le niveau le plus agrégé des qualifications de l'emploi, soit trois catégories : les cadres (dont professions intellectuelles supérieures), les techniciens et professions intermédiaires, les employés-ouvriers ${ }^{5}$. La mobilité spatiale des jeunes est envisagée comme un changement de Zone d'Emploi ${ }^{6}$ entre la résidence

\footnotetext{
${ }^{5} \mathrm{Au}$ sein des cadres, techniciens et professions intermédiaires, des découpages catégoriels changent assez peu les constats d'ensemble, et s'il existe des disparités au sein des catégories employésouvriers, elles ont fait l'objet d'un examen détaillé auquel le lecteur peut se reporter (Lemistre et Magrini, 2008). Les disparités constatées ne remettent évidemment pas en cause l'étude comparative réalisée ici, qui s'enrichit en outre de nombreux éléments d'analyse.

${ }^{6}$ Selon l'INSEE, « une zone d'emploi est un espace géographique à l'intérieur duquel la plupart des actifs résident et travaillent »; elle permet de constituer « une partition du territoire adaptée aux études locales sur l'emploi et son environnement $»$.
} 
de fin des études et le lieu de l'emploi occupé trois ans après la sortie de formation initiale.

L'échantillon retenu, extrait de l'enquête Génération 98 du Céreq, est constitué de 44327 jeunes hommes et femmes, sortis du système éducatif en 1998, et en situation d'emploi en 2001, en France métropolitaine? Dans ce repérage de la mobilité, la distance permet de préciser l'hétérogénéité des comportements de mobilité et l'arbitrage coûts/bénéfices de la décision de mobilité.

Notons que pour l'échantillon retenu, le lien entre le niveau d'études à la sortie du système éducatif et la qualification de l'emploi, trois ans plus tard, s'avère, comme cela a été évoqué plus haut, relativement lâche. Par exemple, les techniciens et professions intermédiaires comptent 5,8\% de bac $+5,17 \%$ de bac +3 ou 4 et, surtout, près d'un quart des employés-ouvriers (23,2 \%) sont diplômés de l'enseignement supérieur $(16 \% \mathrm{bac}+2 ; 5,8 \% \mathrm{bac}+3 / 4 ; 1,4 \% \mathrm{bac}+5)$.

L'observation du dernier emploi occupé, trois ans après la sortie du système de formation initiale, a été privilégiée car il est plus proche d'une forme de stabilisation dans l'emploi que le premier emploi. D'ailleurs, les deux tiers des jeunes déclarent être satisfaits de cet emploi et ne pas être à la recherche d'un nouvel emploi. Ensuite, l'étude des mobilités spatiales montre que les mobilités sont plus nombreuses à l'issue du premier emploi qu'au moment de la prise du premier emploi. Ce constat laisse entrevoir que le jeune élargit progressivement son champ spatial de prospection. En particulier, le premier emploi peut n'être qu'un emploi d'attente dans la poursuite de son effort de prospection sur le marché du travail. Après une ou plusieurs expériences, le jeune acquiert une meilleure connaissance de ses compétences et du marché du travail et peut donc sélectionner plus facilement un meilleur emploi (Johnson, 1978).

La première partie explique le repérage de la mobilité, qui prend en compte la distance kilométrique parcourue entre le lieu d'études et le lieu d'emploi trois ans plus tard. La suite de la partie expose les

\footnotetext{
7 Plusieurs arbitrages ont conduit à retirer les jeunes qui séjournaient à l'étranger, soit à la fin de leurs études, soit pour leur emploi. De même, les jeunes de Corse n'ont pas été pris en compte. Leur mobilité spatiale est susceptible d'être différente des autres jeunes français en raison de la barrière naturelle introduite par la mer.
}

principaux déterminants influençant cette mobilité géographique selon les caractéristiques de l'individu et des territoires. La partie 2 présente les résultats d'une évaluation du rendement salarial de ces mobilités. Les résultats sont examinés dans le cadre de la théorie économique de la prospection d'emploi spatialisée (job search). La mobilité est ici la conséquence d'un arbitrage où le salaire obtenu est confronté à deux types de coûts : les coûts de prospection d'emploi (recherche d'emploi), auxquels s'ajoutent les coûts de mobilité (transport, logement, perte de réseaux sociaux, etc.). Les résultats déclinés par catégorie d'emploi cible conduisent à s'interroger sur deux enjeux de la politique publique : celui de la décentralisation des financements et de l'organisation des formations, et celui des aides publiques à la mobilité pour l'emploi.

\section{L'AMPLITUDE DE LA MOBILITÉ GÉOGRAPHIQUE ET SES DÉTERMINANTS}

La Zone d'Emploi est une aire de mobilité plus petite que celles utilisées dans de nombreuses études (régions, départements). De plus, la prise en compte de la distance permet de décrire plus précisément les mobilités. La première sous-partie décrit, par type d'emploi cible, les mobilités individuelles et leur ampleur. Les déterminants des distances parcourues sont ensuite examinés successivement à travers le rôle des caractéristiques individuelles, puis des caractéristiques des territoires de départ et d'arrivée.

\section{Repérer la mobilité géographique au regard de l'arbitrage distance/revenu}

L'usage très limité de la distance dans l'analyse des mobilités géographiques peut s'expliquer par la fréquente indisponibilité de cette information. Or, sa prise en compte, associée à une aire de mobilité réduite, modifie considérablement les observations par type de qualification. Ainsi, alors que la mobilité géographique est souvent considérée comme une caractéristique des plus qualifiés, le changement de zone 
d'emploi concerne non seulement plus de la moitié des professions intermédiaires (professions intermédiaires $51 \%$, techniciens $57 \%$ ) et cadres (68\%), mais aussi plus du tiers des employés et ouvriers. Selon le niveau de qualification, cela varie d'un tiers pour les moins qualifiés (ouvriers et employés non qualifiés) à $45 \%$ pour les employés qualifiés $(38 \%$ pour les ouvriers qualifiés). Ces mobilités apparaissent très supérieures à celles constatées entre régions, particulièrement pour les moins qualifiés. Ainsi, pour les cadres et professions intellectuelles, le changement d'aire de mobilité multiplie le nombre de mobilités par 1,9 (68\% de changement de zone d'emploi contre $36 \%$ de changement de région) et par près de 3 pour les ouvriers non qualifiés, dont un tiers changent de zone d'emploi alors que seulement $12 \%$ changent de région (coefficient entre 2,4 et 2,7 pour les autres catégories ouvriers-employés).

La prise en compte de la distance affine ces observations. Il s'agit de la distance entre la commune de résidence à la fin des études et celle du lieu de travail trois ans plus tard. ${ }^{8}$ En d'autres termes, seules les distances

${ }^{8}$ Elle a été calculée " à vol d'oiseau » entre les communes de départ et d'arrivée et associée à un changement de zone d'emploi. donnant lieu à changement de zone d'emploi lors de la mobilité géographique ont été retenues.

Il est important de noter que la mesure de l'ampleur de la mobilité pourrait être affinée, en prenant en compte, par exemple, les temps de transport et la possibilité de bénéficier de transports en commun. Les données disponibles ne nous ont pas permis d'atteindre une telle finesse. Néanmoins, de par la définition des zones d'emplois (cf. supra), retenir les mobilités entre ces espaces permet de limiter ce biais lié aux temps et infrastructures de transport. En effet, les zones d'emploi (ZE) apparaissent comme un découpage pertinent pour rendre compte des marchés locaux du travail. Beaumert (1992) rappelle qu'un marché local du travail se définit comme une zone géographique à l'intérieur de laquelle l'individu peut saisir une offre d'emploi sans avoir à changer de lieu de résidence; ou encore du point de vue de l'entreprise, comme la zone géographique regroupant les travailleurs potentiels que l'entrepreneur peut attirer en premier.

La prise en compte de la distance pourrait relativiser les résultats précédents car toutes les mobilités ne sont pas associées au même effort de mobilité. Un individu peut parcourir une très courte distance et être assimilé à une personne mobile parce qu'il franchit

Tableau 1

Distances parcourues entre lieu d'études en 1998 et lieu d'emploi en 2001 lors du changement de zone d'emploi

\begin{tabular}{|c|c|c|c|c|c|c|c|}
\hline & $\begin{array}{c}\text { moyenne } \\
\text { en } \mathrm{km}\end{array}$ & $\begin{array}{c}\text { médiane } \\
\text { en } \mathrm{km}\end{array}$ & $\begin{array}{c}\text { moins de } \\
20 \mathrm{~km}\end{array}$ & $\begin{array}{c}\text { entre 20 } \\
\text { et } 50 \mathrm{~km}\end{array}$ & $\begin{array}{c}\text { entre 50 } \\
\text { ef } 100 \mathrm{~km}\end{array}$ & $\begin{array}{c}\text { entre } 100 \\
\text { et } 300 \mathrm{~km}\end{array}$ & $\begin{array}{c}\text { plus de } \\
300 \mathrm{~km}\end{array}$ \\
\hline Cadres ${ }^{11}$ & 205 & 117 & $19 \%$ & $17 \%$ & $10 \%$ & $24 \%$ & $30 \%$ \\
\hline Professions Intermédiaires & 156 & 68 & $18 \%$ & $25 \%$ & $17 \%$ & $20 \%$ & $20 \%$ \\
\hline Techniciens & 146 & 68 & $18 \%$ & $25 \%$ & $15 \%$ & $24 \%$ & $18 \%$ \\
\hline Employés Qualifiés & 150 & 54 & $23 \%$ & $24 \%$ & $14 \%$ & $20 \%$ & $19 \%$ \\
\hline Ouvriers Qualifiés & 134 & 41 & $28 \%$ & $27 \%$ & $12 \%$ & $15 \%$ & $18 \%$ \\
\hline Employés Non Qualifiés & 151 & 51 & $25 \%$ & $25 \%$ & $12 \%$ & $19 \%$ & $19 \%$ \\
\hline Ouvriers Non Qualifiés & 127 & 37 & $31 \%$ & $27 \%$ & $12 \%$ & $13 \%$ & $17 \%$ \\
\hline TOUS & 158 & 61 & $21 \%$ & $24 \%$ & $14 \%$ & $20 \%$ & $21 \%$ \\
\hline
\end{tabular}

Source : enquêtes génération 98 - Calcul des auteurs.

Lecture : Ce tableau indique les distances parcourues moyennes et médianes et la répartition en pourcentages de ces mobilités pour différents intervalles de distance.

${ }^{(1)}$ Les cadres parcourent en moyenne $205 \mathrm{~km}$ lors des changements de zone d'emploi. $50 \%$ d'entre eux parcourent moins de $117 \mathrm{~km}$ et $50 \%$ plus. L'écart positif entre moyenne et médiane (205 - 117) traduit le fait que certains d'entre eux parcourent de très longues distances, alors que la majorité parcourt de courtes distances. Par exemple, $30 \%$ parcourent plus de $300 \mathrm{~km}$ et $36 \%$ moins de $50 \mathrm{~km}$ (dont $19 \%$ moins de $20 \mathrm{~km}$, $17 \%$ moins de $50 \mathrm{~km})$. 
une frontière entre zones d'emploi tout en parcourant une très courte distance, tandis qu'un autre parcourt une distance dix fois supérieure pour changer de zone d'emploi. Ces différences sont d'autant plus marquées que l'on considère une échelle infrarégionale comme la zone d'emploi. De fait, dans le cas des changements de région, à peine $3 \%$ des mobilités correspondent à des distances de moins de $20 \mathrm{~km}$, tandis que cette proportion s'élève à $21 \%$ dans le cas des changements de zone d'emploi (tableau 1). Or, une telle distance peut être assimilée à un simple franchissement de frontière entre zones d'emploi. En outre, les mobilités inférieures à $20 \mathrm{~km}$ sont proches des déplacements de type domicile/travail ${ }^{9}$. Les déplacements inférieurs à $20 \mathrm{~km}$ ont donc été assimilés à une non-mobilité dans la suite de cet article.

Si les mobilités de moins de 20 km des employésouvriers s'avèrent effectivement plus conséquentes que pour les cadres ( 23 à $31 \%$ des mobilités employésouvriers contre $19 \%$ pour les cadres - tableau 1), les moins qualifiés ne sont pas moins nombreux à parcourir de grandes distances. À titre d'exemple, la proportion de personnes mobiles parcourant plus de $300 \mathrm{~km}$ est tout aussi importante chez les ouvriers non qualifiés que chez les ouvriers qualifiés ou les techniciens. La prise en compte de la distance permet ainsi de relativiser certaines idées reçues sur les capacités de mobilité des différentes catégories de travailleurs.

\section{De l'influence des caractéristiques individuelles}

Un inconvénient majeur des estimations qui ignorent la distance est de supposer un coût uniforme de la mobilité. En effet, pour nombre d'économistes, la distance parcourue est étroitement liée aux coûts de mobilité subis. Il s'agit non seulement des coûts de transport liés à la mobilité ${ }^{10}$, mais aussi du coût psychologique d'éloignement de son capital social et de l'environnement d'origine (Sjaastad, 1962 ; Greenwood, 1997). Dès lors, dans cette approche, la décision de mobilité est prise si les bénéfices associés

\footnotetext{
$975 \%$ des jeunes en emploi en 2001 parcourent quotidiennement une distance de moins de $20 \mathrm{~km}$ pour rejoindre leur lieu de travail (« à vol d'oiseau »).

10 Combes et Lafourcade (2005) montrent une corrélation proche de 1 entre les coûts de transport et la distance géographique en France.
}

à cette décision permettent au minimum de couvrir les coûts de mobilité.

Concrètement, sur le plan empirique, la décision de mobilité est souvent analysée à partir de l'estimation de la probabilité d'être mobile (modèle dit logistique). Cette mobilité estimée est issue d'une approche binaire de la mobilité : la variable de mobilité est codée 1 si le salarié est mobile (au mieux entre zones d'emploi), 0 sinon. La considération conjointe de la mobilité et de la distance parcourue conduit à employer un autre type d'estimation, où la variable de mobilité est considérée comme une variable dite censurée : la variable mobilité prend la valeur 0 pour les non-mobiles entre zones d'emploi (censure) et la valeur de la distance parcourue pour les mobiles (ayant une distance supérieure à $20 \mathrm{~km}$ selon la convention explicitée plus haut). Le modèle estimé est un tobit ; cette procédure permet d'analyser les déterminants des distances parcourues en prenant en compte la probabilité de mobilité (tableau 2) ${ }^{11}$.

Concernant le choix des variables permettant d'expliquer la mobilité géographique, les caractéristiques retenues dans de nombreuses études, anglo-saxonnes principalement, sont les variables personnelles et familiales de l'individu au « départ de sa décision ». Ce sont ces variables qui sont commentées dans cette sous-partie.

Toutes choses égales par ailleurs, les femmes sont moins mobiles, quelle que soit la profession considérée. De même, l'âge réduit la mobilité à niveaux d'études équivalents. Si l'influence du niveau d'études au sein des professions se maintient, les différences de propension à la mobilité entre niveaux d'études se resserrent au sein des professions cibles, voire deviennent non significatives. Par exemple, pour les cadres, au-delà de bac +2 , le niveau d'études ne joue pas différemment entre les bac $+3,4$ et 5 , alors qu'une investigation par niveau d'études révèle

\footnotetext{
11 La distance est prise en compte sous forme logarithmique. Ainsi, faire 500 ou $1000 \mathrm{~km}$ s'explique d'une manière assez similaire, au regard des variables retenues, contrairement à une distance de $50 \mathrm{~km}$ par rapport à $500 \mathrm{~km}$. Cette forme logarithmique traduit des rendements marginaux décroissants de la distance parcourue, soit par exemple le fait qu'un kilomètre de distance supplémentaire au-delà de $300 \mathrm{~km}$ a un effet moyen sur le salaire très inférieur à une même augmentation appliquée à une distance de $30 \mathrm{~km}$ (Da Vanzo, op. cit.).
} 
Tableau 2

Déterminants de la mobilité par qualification

\begin{tabular}{|c|c|c|c|c|}
\hline & TOUS'(1) & Cadres & $\begin{array}{l}\text { Techniciens } \\
\text { - Professions } \\
\text { Intermédiaires } \\
\end{array}$ & $\begin{array}{l}\text { Employés } \\
\text { Ouvriers }\end{array}$ \\
\hline \multicolumn{5}{|l|}{ VARIABLES INDIVIDUELLES } \\
\hline Femme versus homme & -- & -- & -- & -- \\
\hline 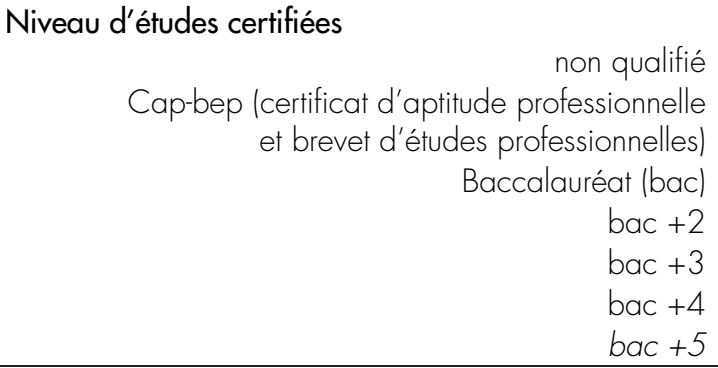 & $\begin{array}{c}---- \\
--- \\
--- \\
--- \\
-- \\
\text { réf. }\end{array}$ & $\begin{array}{c}---- \\
---- \\
\text { ns } \\
\text { ns } \\
\text { réf. }\end{array}$ & $\begin{array}{c}--- \\
--- \\
-- \\
-- \\
\text { réf. }\end{array}$ & $\begin{array}{l}--- \\
-- \\
--\end{array}$ \\
\hline Âge en 98 & -- & --- & -- & - \\
\hline $\begin{array}{l}\text { Situation familiale } \\
\qquad \begin{array}{r}\text { niveau d'études conjoint } \\
\text { niveau d'études conjointe } \\
\text { nombre d'enfants }\end{array}\end{array}$ & $\begin{array}{c}+ \\
++ \\
--\end{array}$ & $\begin{array}{l}\text { ns } \\
\text { ns } \\
--\end{array}$ & $\begin{array}{l}\text { ns } \\
+ \\
--\end{array}$ & $\begin{array}{c}++ \\
++ \\
--\end{array}$ \\
\hline \multicolumn{5}{|l|}{ VARIABLES GÉOGRAPHIQUES } \\
\hline ZE fin études $=\mathrm{ZE} 6^{\mathrm{e}}$ & --- & -- & --- & $-\cdots$ \\
\hline \multicolumn{5}{|l|}{ Pôle d'études 98} \\
\hline pôle urbain & réf. & réf. & réf. & réf. \\
\hline pôle péri-urbain & ++ & ++ & ++ & ++ \\
\hline pôle multipolarisé & ++ & +++ & ++ & ++ \\
\hline pôle rural & ++ & ++ & ++ & +++ \\
\hline \multicolumn{5}{|l|}{$\begin{array}{l}\text { Variable d'écart zones d'emplois 01/98 (2): } \\
\quad \text { (valeur zone d'emploi 2001) - } \\
\quad \text { (valeur pour zone d'emploi fin d'études 98) }\end{array}$} \\
\hline Écart de densité démographique $\times 10^{-4}$ & +++ & ++ & +++ & ++++ \\
\hline Écart des taux de chômage & -- & -- & -- & -- \\
\hline Écart des taux d'études & ---- & ---- & ---- & +++++ \\
\hline $\begin{array}{r}\text { Écart des taux de croissance de la population } \\
\text { dû au solde migratoire }\end{array}$ & - & -- & - & ns \\
\hline
\end{tabular}

Sources : Enquêtes génération 98 - Estimations des auteurs.

Lecture : Les coefficients du modèle Tobit estimé n'étant pas directement interprétables, la lecture des résultats dans les tableaux suivants est simplifiée à l'aide des signes «+ » et « - » qui indiquent l'intensité de l'influence du paramètre considéré sur la distance parcourue pour tous les coefficients statistiquement significatifs au seuil de $10 \%$. «ns » indique que le coefficient n'était pas significatif à ce seuil.

(1) Pour l'ensemble de la population : être une femme diminue les distances parcourues. L'ampleur des différences femmes/hommes est comparable à celle entre niveaux d'études pour un bac +4 par rapport à un bac +5 (ref.). Être diplômé de niveau bac a un impact négatif sur la distance parcourue deux fois supérieur à bac +4 par rapport à la référence $(\mathrm{bac}+5)$.

Plus la densité démographique est forte dans la zone d'emploi où l'individu a migré pour travailler en 2001 par rapport à celle de la zone d'emploi où il a étudié, plus la distance parcourue est importante. À l'inverse, plus le taux de chômage est élevé dans la zone d'emploi 2001, par rapport à la zone d'emploi d'études, plus la distance parcourue est faible.

(2) Les variables d'écarts ont été collectées à partir des données par zone d'emploi du recensement 1999. L'écart du taux de chômage compare le taux de chômage dans la zone d'emploi d'arrivée par rapport à celui de la zone de départ à partir de cette source. L'écart de densité démographique concerne le nombre d'habitants au kilomètre carré. L'écart de taux d'études s'appuie sur la part d'étudiants dans la population des zones d'emploi. Enfin, l'écart de taux de croissance de la population concerne la croissance des actifs due au seul solde migratoire entre les deux recensements généraux de la population de 1999 et 1990 (nombre de travailleurs arrivant dans la zone d'emploi - nombre de partants), ceci pour les zones d'emplois 2001 et 1998. Ces écarts sont évidemment nuls pour les sédentaires.

Variables non reproduites : catégories socioprofessionnelles du père et de la mère en 1998, situations du père et de la mère (chômage, inactif, retraité, privé, public) et enfin la nationalité des parents (français, français par acquisition, étranger). 
des comportements migratoires différents (Magrini, 2006), sans distinguer néanmoins les emplois cibles.

Du côté de la situation familiale, le niveau d'études du conjoint ou de la conjointe n'a pas d'impact pour les cadres. Pour les techniciens, professions intermédiaires et employés-ouvriers, le niveau d'études de la conjointe renforce la mobilité, particulièrement pour les employés-ouvriers. Et seulement pour cette dernière catégorie, le niveau d'études du conjoint renforce la mobilité des femmes, qui est sans doute contrainte par la profession plus qualifiée du mari.

En revanche, pour les cadres, le niveau d'études des conjoints peut, dans certains cas, favoriser la mobilité et, dans d'autres situations, la contraindre, car des logiques de carrière des conjoints peuvent entrer en conflit. Nous pouvons supposer que l'opposition de ces deux cas rend globalement l'effet de cette variable non significatif pour les plus qualifiés.

Quant au nombre d'enfants, il réduit la mobilité de tous les actifs, les enfants pouvant freiner la décision de mobilité pour diverses raisons (changement d'école, coûts de mobilité plus élevés, éloignement de la famille, etc.)

Concernant l'influence de l'héritage social transmis par les parents sur la mobilité de leurs enfants, si pour l'ensemble de l'échantillon la plupart des coefficients de ces variables sont non significatifs (de ce fait non reproduits), les estimations par groupes socioprofessionnels révèlent quelques résultats probants ${ }^{12}$. Pour autant, ils ne seront pas reproduits ici, une analyse plus détaillée étant proposée par Lemistre et Moreau (2008).

\section{De l'influence des variables territoriales sur la mobilité}

Nos investigations proposent également d'observer une influence non négligeable d'autres caractéristiques liées aux territoires et qui sont rarement prises en compte dans la littérature, surtout pour les analyses concernant la France.

\footnotetext{
12 L'absence de prise en compte simultanée de l'influence de ces variables sur la mobilité et le niveau d'études, compte tenu de contraintes techniques, limite l'influence de ces variables. Pour une étude de cette influence conjointe pour la mobilité au premier emploi, voir Lemistre et Moreau (2008).
}

La première variable géographique étudiée concerne la mobilité antérieure, ou plutôt l'absence de mobilité entre la zone d'emploi ou résidait le jeune lorsqu'il était en $6^{\text {e }}$ et la zone d'emploi de fin d'études. Cette sédentarité durant la formation réduit la mobilité, et ce d'autant plus que la qualification de l'emploi diminue. On retrouve ici un résultat fréquent : la mobilité conduit à la mobilité ou l'immobilité à l'immobilité.

Pour autant, cette tendance générale n'exclut pas la mobilité en fonction des caractéristiques du territoire de départ. Ainsi, le fait de résider en milieu urbain à la fin des études limite la mobilité, compte tenu d'une offre d'emploi locale relativement conséquente ${ }^{13}$.

Concernant les variables d'écart, l'ampleur de la mobilité croît avec l'augmentation de la densité de la zone d'emploi, particulièrement pour les employésouvriers ; viennent ensuite les techniciens et professions intermédiaires, puis les cadres. Sachant que les difficultés d'emploi sont plus fortes pour les employésouvriers que pour les autres catégories plus qualifiées, le fait que ces jeunes mobiles soient fortement attirés par des zones d'emploi plus denses peut être interprété comme une forme de « sécurisation » de la stratégie de mobilité. En effet, en se déplaçant vers des zones d'emploi plus denses, ces jeunes augmentent la probabilité de pouvoir trouver un autre emploi.

L'écart des taux de chômage entre zones de départ et d'arrivée apparaît très significatif. Ce résultat suggère que c'est avant tout l'existence d'un « différentiel territorial » qui motive la mobilité. Il montre également que les personnes mobiles privilégient des zones d'emploi plus dynamiques, particulièrement les cadres. Pour l'indicateur d'écart de « taux d'études» (différences dans la proportion d'étudiants entre zones d'emploi 2001 et de fin d'études), la mobilité des employés-ouvriers est renforcée si le taux d'études de la zone d'emploi rejointe est plus élevé que celui de la zone d'emploi de départ. Le phénomène inverse est observé pour les autres groupes de jeunes. Ces derniers ne se dirigent pas majoritairement vers les zones d'emploi disposant d'autres formés qui créent

\footnotetext{
13 A contrario, les ruraux sont plus mobiles, surtout parmi les ouvriers-employés. Pour les autres caractéristiques du territoire, leur forte variabilité entre les zones de départ et d'arrivée nous conduit à préférer l'usage de variables d'écart. Ces dernières rendent mieux compte du différentiel territorial dans le processus de mobilité considéré comme un arbitrage coûts/bénéfices.
} 
une certaine concurrence dans l'emploi. Peut-être pouvons-nous compléter cette explication par le fait que les personnes mobiles vers les emplois les moins qualifiés rejoignent des zones d'emploi où le taux élevé de formés indique a priori une forte proportion d'emplois qualifiés. Or, la présence de ces derniers nécessite celle d'employés-ouvriers dans les mêmes entreprises ou les réseaux de sous-traitants, ou encore pour les services aux particuliers à destination des très qualifiés. Il a été d'ailleurs observé que les « services aux particuliers » généraient plus de mobilité chez les employés-ouvriers (Lemistre et Magrini, 2008). Une autre explication est liée à la présence de surdiplômés parmi les employés et ouvriers qui peuvent aussi rejoindre des zones d'emploi où les emplois qualifiés sont nombreux, en espérant y accéder plus tard. Cela pourrait aussi expliquer pourquoi ces populations se dirigent davantage vers les zones plus denses en population.

Les études sur la mobilité géographique mettent généralement en avant un phénomène de "push and pull » (« répulsion et attraction ») lié aux mobilités antérieures sur le territoire (Greenwood, op. cit.) : un territoire ayant des taux d'immigration géographique antérieurs élevés tend à attirer encore plus de mobiles d'autres territoires. Or, ce résultat ne se confirme pas ici. On peut supposer, et particulièrement pour les cadres, que les zones d'emploi à fort taux d'immigration doivent plutôt attirer un nombre important de travailleurs plus expérimentés avec lesquels les jeunes formés sont en concurrence directe sur le marché du travail. Les plus jeunes choisissent donc des zones d'emploi moins prisées par ces cadres plus expérimentés.

L'effet des variables individuelles et territoriales sur les distances parcourues entre zones d'emploi a été commenté principalement en regard des avantages économiques de la mobilité. Les investigations précédentes s'inspirent en effet des travaux d'économistes qui analysent la mobilité géographique comme le résultat d'un arbitrage coûts/bénéfices, arbitrage qui prend tout son sens dès lors que l'on examine l'impact salarial de la mobilité. La partie suivante tentera une courte synthèse des éléments théoriques de base du domaine et de leur incidence empirique, avant de présenter les résultats.

\section{COÛTS ET BÉNÉFICES DE LA MOBILITÉ EN TERMES DE SALAIRE}

\author{
Quelques éléments d'analyse \\ théorique et empirique
}

La quête de meilleurs revenus est toujours apparue, pour les économistes, comme un motif essentiel de la décision de mobilité (Hicks, 1932 ; Makover et al., 1940 ; Sjaastad 1962 ; Greenwood, 1997 ; Margirier, 2006). Toutefois, pour saisir le sens des écarts de salaire entre personnes mobiles et non mobiles, plusieurs difficultés majeures apparaissent. Leur analyse est toujours faite dans le cadre de la théorie de la quête d'emploi (job search) dont s'inspirent nos investigations. Nous tentons ici de proposer une synthèse, non formalisée, de l'ensemble des problèmes empiriques et théoriques soulevés par ce cadre d'analyse ${ }^{14}$.

La première difficulté est celle de savoir si l'amélioration de la situation d'emploi de la personne mobile est liée à la saisie d'une opportunité salariale préexistante (ex-ante); ou si l'individu mobile dispose de caractéristiques particulières qui créent, après mobilité (ex-post), une meilleure rémunération dans l'emploi par rapport aux individus sédentaires (Nakosteen et Zimmer, 1980). En d'autres termes, est-ce que les personnes mobiles sont les plus « performantes » sur le marché du travail à diplôme et parcours donnés (toutes choses égales par ailleurs) ? Si oui, le choix de mobilité de la majorité d'entre eux révèle alors une plus grande motivation, habileté ou capacité d'adaptation, créant un effet de sélection (ou d'auto-sélection). Dans ce cas, leur salaire relatif plus élevé est dû davantage à leurs qualités intrinsèques qu'à la mobilité elle-même, c'est «l'effet de sélection positif ».

Ce dernier peut néanmoins être négatif. En effet, les plus « performants » peuvent aussi saisir les meilleures opportunités locales, auquel cas les personnes mobiles sont contraintes à la mobilité. Ont-elles pour autant des salaires relatifs moins élevés que les sédentaires? À ce stade de notre exposé, on serait tenté de répondre par l'affirmative. Toutefois, ce serait oublier que les individus ne sont mobiles que si deux types de

\footnotetext{
${ }^{14}$ Voir Lemistre et Magrini (op. cit.) et Magrini (2006) pour une synthèse détaillée des différents éléments repris ici.
} 
coûts sont couverts : les coûts de prospection ou de recherche d'emploi et les coûts de mobilité.

De nombreuses études empiriques confirment que l'impact des coûts de prospection sur le salaire est indéterminé, au sens où il peut être positif ou négatif. En effet, si la mobilité doit compenser les coûts de prospection sur le plan salarial, ces coûts augmentent avec le temps, de telle sorte que l'individu sera prêt à diminuer ses exigences salariales pour compenser cette charge croissante (hypothèse de base du modèle de quête d'emploi « job search »). La compensation des coûts de prospection associée à la diminution des exigences salariales avec le temps peuvent alors expliquer les divergences de résultats selon les durées de recherche d'emploi, les emplois cibles ou encore les caractéristiques des territoires (pour une synthèse sur ce point, $c f$. Drapier et alii, 2004).

Pour les coûts de mobilité (déménagement, logement, perte de réseaux sociaux, pertes d'aménités, etc.), nul doute que ces derniers doivent être compensés si l'on suppose une prise en charge individuelle (Molho, 1986). Ainsi, même si les personnes mobiles ne sont pas parmi les plus «performantes », leurs salaires relatifs par rapport aux sédentaires peuvent être plus élevés en compensation des coûts de mobilité. En d'autres termes, leur salaire élevé ne traduit pas un « gain net » par rapport aux sédentaires, ou encore une situation financière plus favorable pour eux après mobilité malgré l'obtention d'un salaire plus élevé (Nakosteen et Zimmer, 1980).

Comme cela a été évoqué plus haut, la très grande majorité des recherches saisissent les effets salariaux de la mobilité en distinguant uniquement entre mobiles et non-mobiles, sans prise en compte de la distance. De plus, la mobilité est généralement considérée entre des aires de grande taille (départementsrégions) et souvent les " effets de frontière » sont négligés (ie. un déplacement d'un kilomètre entre deux régions est considéré comme une mobilité interrégionale, $c f$. supra). Enfin et surtout, une des principales limites empiriques de cette méthode est de ne saisir qu'un effet moyen de la mobilité qui cumule l'effet des caractéristiques individuelles intrinsèques avec ceux des coûts de prospection et de mobilité.

La distance permet de saisir plus précisément l'effet des coûts de mobilité, sans pour autant lever l'indéter- mination quant à l'impact des coûts de prospection. En effet, le lien entre la distance parcourue lors de la mobilité (ex post) et les coûts subis pendant la prospection ne va pas de soi. Par exemple, un individu peut avoir été mobile à une courte distance in fine, alors qu'il avait prospecté longtemps et très loin avant de réaliser cette courte mobilité. La proportionnalité entre coût de prospection et distance n'est donc pas évidente.

En tout état de cause, la distance permet de mieux séparer l'effet des coûts de mobilité de l'effet de sélection lors des estimations. Sans entrer dans les détails techniques, il est essentiel ici d'expliciter les trois estimations effectuées. La première est une régression par les moindres carrés ordinaires mettant en relation le salaire (variable expliquée) et la distance parcourue (variable explicative) entre la fin d'études en 1998 et l'emploi 2001, toutes choses égales par ailleurs, soit avec comme autres variables explicatives du salaire : des caractéristiques individuelles (sexe, niveau d'études, âge, parcours d'insertion), des caractéristiques liées à l'emploi (taille de l'entreprise, secteur privé/public, contrat de travail, fonction exercée, secteur d'activité), des déterminants du contexte territorial d'emploi (tels que des caractéristiques du pôle d'emploi : urbain, rural, etc., $c f$. tableau 2) et la région d'emploi ${ }^{15}$.

Pour cette estimation, le rendement de la mobilité estimé est une moyenne qui reflète le surplus salarial moyen des personnes mobiles : rendement moyen (tableau 3). La variable capte les effets joints des coûts de prospection, de mobilité et des caractéristiques intrinsèques (effet de sélection). Rappelons que si la grande majorité des personnes mobiles sont les plus performantes toutes choses égales par ailleurs (à diplôme, parcours, catégories d'emploi égaux), le coefficient de la variable mobilité traduit alors le surplus salarial des « meilleurs » travailleurs et non un effet propre de la mobilité. En d'autres termes, la variable mobilité capte une partie des caractéristiques inobservées sans que son coefficient soit directement associé aux bénéfices de la seule mobilité. Pour saisir ce dernier

\footnotetext{
${ }^{15}$ L'équation d'estimation est une fonction de gains du type : $\ln ($ salaire $)=\mathrm{a}+\mathrm{b} \ln ($ distance $)+\mathrm{c}($ sexe $)+\mathrm{d}$ (pôle d'emploi $)+\mathrm{f}$ (régions d'emploi) $+\mathrm{g}$ (niveau d'études) $+\mathrm{f}($ âge $)+$ l'ensemble des autres variables explicatives exposées dans le texte. Le détail des estimations est à la disposition des lecteurs auprès des auteurs. Des estimations avec le même type de variables explicatives figurent dans Magrini (2006).
} 
Tableau 3

Gain salarial en \% selon la distance parcourue

entre la sortie du système éducatif en 1998 et l'emploi de 2001

\begin{tabular}{|c|c|c|c|c|c|c|}
\hline Catégories & $\begin{array}{l}\text { Distances parcourues } \\
\text { Rendement salarial }\end{array}$ & $50 \mathrm{~km}$ & $100 \mathrm{~km}$ & $300 \mathrm{~km}$ & $600 \mathrm{~km}$ & $900 \mathrm{~km}$ \\
\hline \multirow{3}{*}{ TOUS } & moyen (moy.) & $2,4 \%$ & $3,0 \%$ & $3,9 \%$ & $4,5 \%$ & $4,7 \%$ \\
\hline & /variables observées (obs.) & $4,9 \%$ & $6,3 \%$ & $8,2 \%$ & $9,3 \%$ & $9,9 \%$ \\
\hline & /variables observées et d'écart 01/98 (obs.ec.) & $2,1 \%$ & $2,7 \%$ & $3,4 \%$ & $3,9 \%$ & $4,1 \%$ \\
\hline \multirow{3}{*}{ Cadres } & moyen (moy.) & $0,7 \%$ & $1,0 \%$ & $1,2 \%$ & $1,4 \%$ & $1,5 \%$ \\
\hline & /variables observées (obs.) & $-7,7 \%$ & $-9,9 \%$ & $-12,5 \%$ & $-14,0 \%$ & $-14,8 \%$ \\
\hline & /variables observées et d'écart 01/98 (obs.ec.) & $-2,0 \%$ & $-2,5 \%$ & $-3,2 \%$ & $-3,6 \%$ & $-3,9 \%$ \\
\hline \multirow{3}{*}{$\begin{array}{c}\text { Techniciens } \\
\text { Professions } \\
\text { intermédiaires }\end{array}$} & moyen (moy.) & $2,3 \%$ & $2,9 \%$ & $3,8 \%$ & $4,3 \%$ & $4,6 \%$ \\
\hline & /variables observées (obs.) & $4,6 \%$ & $5,9 \%$ & $7,7 \%$ & $8,7 \%$ & $9,3 \%$ \\
\hline & /variables observées et d'écart 01/98 (obs.ec.) & $2,4 \%$ & $3,1 \%$ & $4,0 \%$ & $4,6 \%$ & $4,9 \%$ \\
\hline \multirow{3}{*}{$\begin{array}{l}\text { Employés - } \\
\text { Ouvriers }\end{array}$} & moyen (moy.) & $2,0 \%$ & $2,6 \%$ & $3,3 \%$ & $3,8 \%$ & $4,0 \%$ \\
\hline & /variables observées (obs.) & $2,4 \%$ & $3,1 \%$ & $4,1 \%$ & $4,6 \%$ & $4,9 \%$ \\
\hline & /variables observées et d'écart 01/98 (obs.ec.) & $2,1 \%$ & $2,8 \%$ & $3,6 \%$ & $4,0 \%$ & $4,3 \%$ \\
\hline
\end{tabular}

Source : enquête génération 98 - estimations des auteurs.

Lecture : Pour les techniciens et professions intermédiaires, «toutes choses observées égales par ailleurs », le rendement moyen d'une mobilité de $100 \mathrm{~km}$ est de 2,9\% de salaire en plus. Si l'on considère uniquement le rendement associé aux caractéristiques observées, il est de 5,9\% (hors caractéristiques inobservées : innées ou associées à des variables non disponibles). Dès lors que l'on prend en compte, parmi les déterminants de la distance parcourue, les écarts entre territoires (taux de chômage notamment, $c f$. tableau 2 " variables d'écart »), le rendement moyen est de 3,1\%, soit un rendement positif de la mobilité, même après compensation des caractéristiques des territoires de départ et d'arrivée.

élément, il est donc nécessaire de séparer l'impact des variables observables et inobservables.

Pour cela, la première étape consiste à construire une variable mobilité dépendant uniquement d'éléments observés. Deux spécifications ont été retenues à cette fin. La première reprend l'ensemble des variables de la régression présentée dans la partie précédente (tableau 2), excepté les variables d'écart entre territoires de formation et lieu d'emploi 2001. Les rendements estimés constituent alors le résultat de la seule mobilité associée aux variables observées. Cet effet salarial lié à la distance est associé principalement aux coûts de mobilité. La procédure mobilisée relève de la méthode des variables instrumentales ${ }^{16}$. En réalité, les économètres ont souvent tendance à confondre observable et observé. En effet, les variables explicatives de la distance parcourue sont les seules variables « disponibles » dans l'enquête mobilisée, mais ne recouvrent

${ }^{16}$ Ici, la régression auxiliaire est un tobit (modèle tronqué à droite : absence de mobilité versus kilomètres parcourus, tableau 2) et la méthode est celle de Nelson et Olson (1978). Voir Lemistre et Magrini (2008) pour le détail. pas toutes les variables « potentiellement observables » et explicatives de la mobilité. Les méthodes demeurent donc largement imparfaites. Dans l'optique où l'inobservé et l'inobservable sont supposés confondus, ce qui n'est pas observé est souvent supposé traduire les compétences « innées » des individus. Or, il s'agit en fait des aptitudes non observées via les variables disponibles. Leur impact positif sur le salaire traduit donc des performances relatives plus élevées des individus meilleurs en regard des variables inobservées, dont les compétences innées peuvent être une composante importante ou négligeable ! Les qualificatifs de « meilleur » ou " plus performant », utilisés dans les développements suivants, sont donc à appréhender en ce sens, c'est-à-dire plus performant toutes choses « observées » égales par ailleurs (diplôme, âge, parcours, territoire, etc.).

\section{Un bénéfice de la mobilité pour rémunérer les plus « performants »?}

Les estimations successives permettent d'obtenir des indicateurs sur la nature des personnes mobiles. Par 
exemple, si le rendement moyen « toutes choses égales par ailleurs » de la mobilité (obtenu par la méthode des moindres carrés ordinaires, $c f$. tableau 3 « moy») est supérieur à celui estimé via la méthode instrumentale qui « purge » l'estimation de l'effet des variables individuelles inobservées ( $c f$. tableau 3 «obs»), cela signifie que tout ou partie du rendement moyen est associé au fait que ce sont les « meilleurs », ou plutôt les plus performants sur le marché du travail (toutes choses égales par ailleurs) qui sont mobiles ${ }^{17}$. Un tel résultat correspond au " biais de sélection positif». Dans le cas opposé, nous sommes en présence d'un « biais de sélection négatif » indiquant que ce ne sont pas les meilleurs qui sont mobiles. Ces deux types d'estimation sont reproduits pour chaque profession dans le tableau 3 : moy et $/$ obs. ${ }^{18}$.

Concernant les rendements moyens calculés pour différentes distances parcourues ( $c f$. tableau 3, lignes moy), les résultats semblent aller dans « le sens commun » où l'effort de mobilité serait «payant» (rendements positifs), et ce d'autant plus que la distance parcourue est importante. Mais le $2^{\mathrm{e}}$ type d'estimation montre que, pour les cadres, ce coefficient de la distance est en fait largement surestimé puisqu'il devient négatif (comparaison des colonnes moy et /obs.). En d'autres termes, la rémunération obtenue au travers de la mobilité ne correspond qu'à l'effet de caractéristiques inobservées jouant favorablement à la fois sur la distance parcourue et sur le salaire. Ces jeunes mobiles peuvent donc être considérés comme des individus «meilleurs » qui, $a$ priori, auraient obtenu de meilleurs salaires même en l'absence de mobilité. En outre, l'absence de rémunération in fine de la mobilité laisse envisager que ces jeunes mobiles sont avant tout confrontés à un marché national du travail. Par conséquent, le passage d'un marché local du travail à un autre ne requiert pas d'amortissement spécifique lié à la mobilité. La personne mobile supporte donc les coûts de la mobilité (d'où les coefficients négatifs de l'estimation / $o b s ., c f$. tableau 3).

\footnotetext{
${ }^{17}$ En d'autres termes, le rendement de la mobilité est « surestimé » par la méthode des moindres carrés ordinaires.

${ }_{18}$ Pour toutes les estimations, la distance parcourue lors du changement de territoire aboutit à un effet largement significatif de cette variable. De même, les tests d'endogénéité sont toujours largement significatifs.
}

$\mathrm{Au}$ contraire, pour les autres qualifications des emplois occupés après mobilité, le rendement moyen de la mobilité s'avère sous-estimé. Ainsi, bien que ces individus aient des caractéristiques défavorables qui les ont probablement contraints à élargir leur champ spatial de recherche d'emploi, ils n'acceptent un emploi extérieur à la zone d'emploi que si celui-ci rémunère en partie ou totalement le coût de la mobilité. Ce résultat laisse donc envisager, à l'opposé des cadres, que ces jeunes travailleurs s'insèrent eux sur des marchés locaux du travail. Le passage d'un marché à l'autre exige de leur part un amortissement spécifique. Notons que les techniciens et professions intermédiaires obtiennent des rendements de la mobilité plus élevés que les employés-ouvriers. Ces différences rendent compte d'un coût de mobilité différencié entre ces professions, mais également de l'étendue plus réduite de la fourchette des rémunérations pour les jeunes de niveau employés-ouvriers.

En termes de gain associé à la mobilité, les rendements de la distance, purgés des inobservés (/obs.), peuvent être comparés aux rendements des diplômes pour évaluer leur ordre de grandeur. Si l'on considère une distance parcourue de $100 \mathrm{~km}$ pour des employés et ouvriers, le rendement de 3,1\% atteint l'écart de rémunération entre un jeune employé-ouvrier sans diplôme et un homologue de niveau bac (toutes choses égales par ailleurs). Ou encore, quelle que soit la distance parcourue, ce rendement compense le gain de deux années d'études après le bac pour les employésouvriers de niveau bac. Du côté des techniciens et professions intermédiaires, le rendement de la même distance est aussi à la hauteur de l'écart entre niveaux d'années d'études. Plus précisément, un rendement de $5,9 \%$ pour $100 \mathrm{~km}$ représente quasiment l'écart de rémunération entre un technicien / profession intermédiaire de niveau bac +3 et un homologue d'un niveau supérieur (bac +4/+5). Au-delà de $100 \mathrm{~km}$, cet écart est dépassé. Ainsi, qu'il s'agisse des employésouvriers ou des techniciens et professions intermédiaires, le rendement salarial de la mobilité est loin d'être négligeable ${ }^{19}$.

\footnotetext{
${ }^{19}$ Les résultats des fonctions de gains où figurent les rendements des niveaux d'éducation par catégorie d'emploi ne sont pas reproduits ; ils sont disponibles auprès des auteurs.
} 


\section{Une mobilité pour compenser les faiblesses du territoire $d^{\prime}$ origine?}

Les bénéfices de la mobilité peuvent être associés à la saisie d'opportunités hors territoire d'origine. Comme nous l'avons vu, la mobilité intervient souvent pour compenser les inconvénients du territoire d'origine (taux de chômage relatif élevé, pas de poste au niveau de qualification souhaité, etc.). Introduire des variables d'écart entre territoires comme variables explicatives de la distance permet d'estimer un rendement de la mobilité indépendant de ces écarts. C'est l'objet de la dernière estimation reproduite dans le tableau 3 (lignes /obs.ec.).

Il est important de noter que l'on intègre rarement des variables «d'arrivée » parmi les variables explicatives de la mobilité et encore moins des variables d'écart. Tout simplement car celles-ci sont susceptibles d'être aussi des variables explicatives du salaire et également d'être expliquées par les déterminants de la mobilité géographique. Ici les variables retenues ( $c f$. tableau 2 « variables d'écart zones d'emplois 01/98 ») sont peu, et le plus souvent pas du tout, explicatives du salaire. En revanche, elles ne sont évidemment pas indépendantes des variables explicatives observées ou non de la mobilité. En clair, la dernière estimation précise uniquement si le différentiel de salaire est le résultat d'une mobilité pour compenser certaines caractéristiques défavorables du territoire d'origine, mais elle n'invalide en rien l'analyse précédente. Des exemples concrets permettent de clarifier le raisonnement.

Ainsi, entre les deux dernières estimations, pour les employés-ouvriers et les techniciens et professions intermédiaires, le rendement diminue ; à l'inverse, pour les cadres et professions intellectuelles, le rendement négatif est très faible. Ces variations indiquent que, pour l'ensemble des professions, la mobilité relève avant tout d'une compensation des inconvénients relatifs du territoire d'origine (voir plus haut l'impact des variables d'écart sur la mobilité). Pour les cadres, ce sont donc les meilleurs (moy < /obs.) qui font preuve de mobilité, mais ils subissent le coût de mobilité (rendement négatif/obs.), d'où un rendement moyen (moy) de la mobilité positif très limité. Quant aux ouvriers, employés, techniciens et professions intermédiaires, si le surplus issu de la mobilité est effectivement associé à la mobilité sur un territoire plus favorable (/obs $>/ o b s+e c$ ), il demeure un rendement moyen élevé de la mobilité qui compense tout ou partie des coûts de mobilité. Prenons un exemple fictif pour clarifier les causalités : un technicien migre vers un autre territoire car les opportunités locales pour ce type d'emploi ont été pourvues par des candidats plus performants (notamment en regard des caractéristiques inobservées). Le territoire visé a des caractéristiques plus favorables (départ d'un territoire rural vers une métropole, par exemple) qui lui permettent d'obtenir un surplus salarial par rapport à un sédentaire aux caractéristiques observables comparables (/obs $>$ moy). Le différentiel s'explique en partie par les opportunités plus nombreuses sur le territoire d'arrivée (/obs </obs+ec). Toutefois, il existe un avantage salarial au-delà du surplus lié au territoire : surplus destiné à compenser, a minima, les coûts de mobilité (/obs+ec $>0$ ).

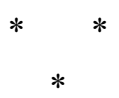

En résumé, le bénéfice de la mobilité des cadres correspond en fait à la rémunération de qualités inobservées favorables. Les jeunes qui recherchent ce type d'emploi sont donc confrontés à un marché national du travail, et, par conséquent, leur effort de mobilité spatiale n'exige pas une compensation des coûts de mobilité en sus de la rémunération de leurs qualités intrinsèques.

Les autres jeunes de profession intermédiaire ou employé-ouvrier obtiennent, au contraire, un rendement positif de la mobilité, malgré l'effet défavorable de caractéristiques inobservées, laissant entrevoir que la mobilité est plus contrainte pour ces jeunes. Les rendements obtenus sont relativement élevés, y compris pour les employés-ouvriers. À la différence des cadres, ces jeunes sont donc confrontés à une multitude de marchés locaux du travail (les zones d'emploi ici) et le passage de l'un à l'autre nécessite une rémunération des coûts de mobilité subis, en fonction de la distance parcourue et de caractéristiques individuelles, familiales et sociales. Ce surplus salarial est toutefois plus ou moins compensé par l'« avantage comparatif» en termes de salaire associé au territoire de destination par rapport au territoire de départ et rend ainsi le rendement de la mobilité variable en fonction des caractéristiques territoriales. 
Les zones d'emploi retenues ici comme unité d'analyse ont été définies au début des années 80 , en vue d'aider les acteurs publics dans l'orientation des politiques de formation au regard de l'emploi local. Face aux choix de mobilité des individus mis en exergue, quels sont alors les enjeux de territorialisation de la politique de l'emploi et de la formation (Berthet et al. 2002) ?

Tout d'abord, le fait que la mobilité soit une réalité pour nombre de jeunes pose la question de savoir à quels marchés du travail la formation dispensée sur d'autres marchés (assimilés à la zone d'emploi, par exemple) profite, et donc, quel partage des responsabilités dans le financement et la définition des formations doit s'opérer entre l'État et les instances territoriales. Au vu des résultats, il semblerait que les formations qui destinent les jeunes à des emplois de cadres doivent relever de l'État, tant dans leur financement que dans leur définition, dans la mesure où le marché du travail de ces jeunes est avant tout national. La mobilité pour ces derniers serait une évidence qui n'induit pas un amortissement des coûts de mobilité. Si l'on suppose que ces emplois sont tous pourvus par des bac +5 , l'identité qualification de l'emploi et niveau de diplôme permettrait aisément d'en tirer les conséquences politiques. Toutefois, pour les jeunes en emploi en 2001, sortis du système éducatif en 1998 , les bac +5 représentent moins de $60 \%$ des cadres et, surtout, plus d'un jeune sur vingt de qualification technicien ou profession intermédiaire est détenteur d'un bac +5 . Or, nos résultats suggèrent que les logiques diffèrent selon la qualification de l'emploi cible, quel que soit le niveau de diplôme du jeune. Ainsi, parmi les bac +5 et les bac +3 et 4 (un cadre sur quatre) certains sont confrontés à un marché national, tandis que d'autres font face à des marchés locaux. Pour les autres niveaux de formation (inférieurs à bac +3 ) qui visent très majoritairement des emplois «non cadres », les logiques restent plus locales. Ainsi, au sein de tous les niveaux de formation, des jeunes accèdent à des emplois appartenant à un marché local. Le financement, même partiel, par les instances locales, est donc justifié pour tous les niveaux de formation.

Actuellement, et suite à la décentralisation, en moyenne, les collectivités territoriales participent à hauteur de $22 \%$ au financement de la formation initiale ${ }^{20}$, mais sans que l'on connaisse le détail de ce financement. Dans l'enseignement supérieur, l'autonomie des universités, en cours de réalisation, va vraisemblablement intensifier l'ancrage local et diversifier les financements, pas seulement au niveau de la recherche. Si tant est que les financements de l'État puissent être davantage affectés à l'avenir, ceux-ci seraient donc davantage justifiés pour les plus hauts niveaux de formation qui relèvent, pour partie, d'une logique de marché national. Sans que cela exclue les financements locaux, y compris au plus haut niveau où certains jeunes s'insèrent sur des marchés locaux « non cadres ». Comme l'accès à la qualification pour un niveau de formation donné dépend largement des spécialités et des filières de formation (Lemistre, 2010), il conviendrait de mieux saisir les différences entre grandes spécialités en regard de la mobilité géographique ; ceci pour assurer une équitable péréquation dans l'affectation des moyens entre les différentes formations, selon que la filière destine très majoritairement les jeunes à s'insérer sur un marché national ou local, par exemple. La péréquation s'entend aussi entre territoires, puisque nos résultats suggèrent que ce sont avant tout les caractéristiques du territoire qui influencent le choix de mobilité. Les quelques variables mobilisées ici constituent alors des pistes de réflexion pour des dotations étatiques plus ou moins concentrées sur certains territoires. En effet, certains territoires peuvent « produire » nombre de bac +5 et en même tant détenir toutes les « caractéristiques territoriales » (taux de chômage relatif faible, densité d'emplois qualifiés forte, etc.) qui vont limiter leur mobilité.

Ensuite, nous pouvons nous interroger sur les aides instituées pour aider ces jeunes dans leur effort de mobilité, notamment parce que les jeunes mobiles vers des postes d'employés-ouvriers ou de professions intermédiaires sont plutôt des individus qui pâtissant de facteurs défavorables à leur insertion qui les conduisent à être mobiles. La mobilité étant chez ces individus plus contrainte, à l'inverse des jeunes cadres, un rendement est escompté pour compenser, en partie ou totalement, les coûts de mobilité subis. Aussi, la mise en place d'aides financières à la mobilité peut aider ces jeunes dans cette décision, notamment pour

\footnotetext{
20 Source : ministère de l'Éducation nationale (http://www.education.gouv.fr/cid28/le-financement-de-1-education.html).
} 
parcourir de plus grandes distances. En France, depuis les années 2000, les aides à la mobilité se sont renforcées, notamment au sein de l'ANPE (Agence nationale pour l'emploi), devenue Pôle Emploi. Aujourd'hui, il existe principalement une prime uniforme à la mobilité géographique qui peut être cumulée avec une aide liée au secteur d'activité d'embauche pour les jeunes. L'aide " Mobilité Professionnelle » permet ainsi de bénéficier d'un crédit d'impôt de $1500 €$ si la prise de l'emploi s'accompagne d'un déménagement de plus de $200 \mathrm{~km}$ par rapport au domicile d'origine. Les jeunes de moins de 26 ans qui vont travailler dans des secteurs de pénurie de main-d'œuvre peuvent bénéficier d'un crédit d'impôt plafonné à $1500 €$ en fonction de leurs revenus. Si on peut louer l'existence de telles aides, les résultats obtenus amènent cependant plusieurs critiques :

D'abord, ces aides devraient dépendre de la qualification de l'emploi cible, d'autant que les possibilités de rendement de la mobilité sont plus réduites pour les emplois moins qualifiés en raison du resserrement des fourchettes de rémunération.

Ensuite, elles devraient aussi être liées à la distance, dès que celle-ci dépasse le seuil nécessitant un déménagement, qui est bien en-deçà des $200 \mathrm{~km}$. Pourquoi ne pas songer à une aide proportionnelle à la distance parcourue plutôt qu'une aide forfaitaire ? Certes, pour les trois-quarts des jeunes mobiles employés et ouvriers étudiés, la mobilité à été inférieure à $200 \mathrm{~km}$ sans, a priori, qu'ils aient eu besoin de cette aide. Mais en revanche, les jeunes qui ne sont pas en emploi l'auraient-ils été s'ils avaient bénéficié d'une aide à la mobilité qui soit en-deçà du seuil des $200 \mathrm{~km}$ ? Précisons ici qu'à la date d'enquête, $48 \%$ des jeunes les moins formés ne sont pas en emploi et que $29 \%$ d'entre eux n'ont connu aucun emploi au cours des trois ans. Pour les jeunes ayant un Cap, Bep/bac, les chiffres sont respectivement de $25 \%$ et $8 \%$. Or, ces jeunes constituent l'essentiel des emplois d'employés et ouvriers. Selon la même logique que la question des financements des formations, on peut s'interroger sur la délégation de ces aides aux Régions : à la région d'origine pour une mobilité intra-régionale, à la région d'accueil pour une mobilité interrégionale.

Enfin, si les choix de mobilité des jeunes s'opèrent en priorité vers des territoires dynamiques, les aides doivent-elles être orientées vers d'autres territoires moins attractifs dans la stratégie de mobilité de ces individus ? Le principe d'associer une aide pour les jeunes qui s'orientent vers des secteurs en pénurie de main-d'œuvre est une bonne chose. Mais ce principe pourrait être complété par un dispositif territorial prenant aussi en compte la dynamique démographique et d'emploi du territoire d'accueil.

\section{Bibliographie}

Beaumert F. (1992), " L'analyse des disparités locales de chômage : objets et enjeux » in Arliaud M., Lamanthe A., Romani C., Les approches du local. Objets, méthodes et itinéraires de recherche, Céreq Document de travail, ${ }^{\circ}$ 76, pp. 43-64.

Beduwé C. et Planas J. (2001), Education Expansion and Labour market, Report European program TSER 4.

Berthet T., Cuntigh P., Guitton C. (2002), « La politique de l'emploi au prisme des territoires »,
Document d'études de la DARES, $\mathrm{n}^{\circ} 56$.

Boumahdi R. et Lemistre P. (2007), « Devenir cadre : une affaire de famille, de genre ou d'éducation ? ", Revue d'économie appliquée, $\mathrm{n}^{\circ} 2$, pp. 123-156.

Combes P. et Lafourcade M. (2005), "Transport Costs: Measures, Determinants, and Regional Policy Implications for France", Journal of Economic Geography, n 5(3), pp. 319-349. 
DaVanzo J. (1983), "Repeat Migration in the United-States : Who moves back and Who moves on ?", Review of Economics and Statistics, vol. 65, pp. 552-559.

Debrand T. et Taffin C. (2005), « Les facteurs structurels et conjoncturels de la mobilité résidentielle depuis 20 ans », Économie et Statistique, n 381-382, pp. 125-146.

Détang-Dessendre C., Drapier C. and Jayet H. (2004), "The Impact of Migration on Wages: Empirical Evidence from French Youth", Journal of Regional Science, Vol. 44, 4, pp. 661-691.

Drapier C. et Jayet H. (2002), « Les migrations des jeunes en phase d'insertion professionnelle en France », Revue d'économie rurale et urbaine, $\mathrm{n}^{\circ} 3$, pp. 355-376.

Drapier C. (2001), Le rôle de la mobilité géographique dans l'insertion professionnelle des jeunes: une application au cas de la France, Thèse de doctorat, université de Lille 1.

Dauty F., Lemistre P., Vincens J. (2006), « Le sens, la portée et le devenir de la nomenclature des niveaux de formations », document CPC.

Freyssinet J. (2004), Le chômage, La Découverte, coll. « Repères ».

Giret J.-F. et Lemistre P. (2004), « Déclassement des jeunes, vers un changement de valeur des diplômes?», Special Issue: "The Economics of Education : unkept promises? ", Brussels Economic Review, vol. 43, pp. 483-504.

Giret J.-F., Lopez A. et Rose J. (2005), « Des formations pour quels emplois ?», La Découverte, coll. « Recherche».

Green A. E., Baldauf B., Owen D. (2009), Study on short-term mobility, rapport pour la Commission européenne $\mathrm{n}^{\circ} \mathrm{VC} / 2008 / 0007$.

Greenwood M.J. (1997), "Internal Migration in Developed Countries" in Handbook of Population and Family Economics, Chapter 12, Vol. 1B, Elsevier Science, pp. 647-720.

Hicks J.R. (1932), The theory of wages, Éditions Macmillan, Londres.

Jayet H. (1993), « Migration, mobilité professionnelle et urbanisation », Dossier de recherche Cesure, $n^{\circ} 1 / 93$.

Johnson W. (1978), “A Theory of Job Shopping”, Quarterly Journal of Economics, vol. 92, pp. 261-277.

Lemistre P. (2010), La formation initiale une valeur sûre pour les jeunes?, Presses de l'université de Toulouse 1 Capitole.

Lemistre P. et Moreau N. (2009), "Spatial mobility and returns of education: some evidence from a sample of French youth", Journal of Regional Sciences, vol. 49, $\mathrm{n}^{\circ} 1$, pp. 149-176.

Lemistre P. et Magrini M.-B. (2008), « La mobilité géographiques des jeunes ouvriers et employés estelle rentable? », Économie et Prévision, n 185.

Long L. (1988), Migration and Residential Mobility in the United States, Russell Sage Foundation, New-York.

Magrini M.-B. (2007), « Les rendements de la mobilité spatiale des jeunes actifs. Une analyse comparative par niveau de formation ", Revue d'économie régionale et urbaine, $\mathrm{n}^{\circ} 4$.

Magrini-Thibault M.-B. (2006), La mobilité géographique des jeunes en insertion professionnelle, Thèse de doctorat, université de Toulouse 1.

Makover H.J., Marschak J., Robinson H.W. (1940), "Studies in mobility of labor: analysis for Great Britain, Part II", Oxford Economic Papers, vol. 4, pp. 39-62.

Margirier G. (2006), « Mobilité géographique et salaires », Revue d'Économie Politique, n ${ }^{\circ} 116(5)$, pp. 657-681.

Molho I. (1986), "Theories of Migration: a Review", Scottish Journal of Political Economy, vol. 33, pp. 396-419. 
Murdoch J. et Perret C. (2007), « Mobilités internationales des diplômés de l'enseignement supérieur français en phase d'insertion professionnelle : déterminants individuels et effets sur le début de carrière lors du retour en France » in Les Migrations Internationales. Observation, analyse et perspectives, Colloque international de Budapest, Hongrie, 20-24 septembre 2004, AIDELF ${ }^{\circ} 12$.

Nakosteen R., Zimmer M. (1980), "Migration and Income: The Question of Self-Selection", Southern Economic Journal, vol. 46, pp. 840-851.

Nelson F. and Olson L. (1978), "Specification and Estimation of a Simultaneous-Equation Model with Limited Dependent Variables", International Economic Review, vol. 19, n 3, pp. 695-709.
Pailhé A. et Solaz A. (2001), « Mobilité géographique, professionnelle et gains salariaux des hommes et des femmes », SESAME, Séminaire d'études et de statistiques appliquées à la modélisation en économie, septembre, Lille.

Perret C. et Roux V. (2004), « La mobilité géographique en début de carrière : un moteur de réussite ? », Formation Emploi n ${ }^{\circ}$ 87, pp. 45-62.

Sjaastad A. L. (1962), "The Costs and Returns of Human Migration”, Journal of Political Economy, vol. suppl. 70, pp. 80-93.

Yankow J. (2003), "Migration, Job change, and wage growth: a new perspective on the pecuniary return to geographic mobility", Journal of Regional Science, vol. 43(3), pp. 486-516.

\section{Résumé}

\section{Mobilité géographique des jeunes : du système éducatif à l'emploi}

Une approche coûts/bénéfices des distances parcourues

Philippe Lemistre et Marie-Benoît Magrini

La mobilité géographique des jeunes actifs est souvent considérée comme un attribut des plus qualifiés. Or, la considération conjointe d'une échelle infrarégionale et de la distance parcourue montre que les jeunes les moins qualifiés peuvent aussi parcourir de grandes distances. Pour autant, les comportements de mobilité restent différenciés selon la qualification et sont également tributaires des caractéristiques territoriales. Cet article propose une lecture économique en considérant la mobilité comme la résultante d'un arbitrage coûts-bénéfices. L'observation des rendements salariaux de la mobilité géographique et de leurs déterminants révèle alors des logiques différenciées selon la qualification de l'emploi cible.

Mots clés :

Mobilité géographique, jeune, salaire, niveau de formation

Journal of Economic Literature: J 62 